\title{
Optimized Adaptive Neuro-Fuzzy Inference System Using Metaheuristic Algorithms: Application of Shield Tunnelling Ground Surface Settlement Prediction
}

\author{
Xinni Liu, ${ }^{1,2}$ Sadaam Hadee Hussein, ${ }^{3}$ Kamarul Hawari Ghazali, ${ }^{2}$ Tran Minh Tung, \\ and Zaher Mundher Yaseen $\mathbb{D}^{4}$ \\ ${ }^{1}$ School of Information, Xi'an University of Finance and Economics, Xi'an, China \\ ${ }^{2}$ Faculty of Electrical and Electronics Engineering Technology, Universiti Malaysia Pahang, Pekan 26600, Malaysia \\ ${ }^{3}$ Almaaref University College, Civil Engineering Department, Ramadi, Iraq \\ ${ }^{4}$ Faculty of Civil Engineering, Ton Duc Thang University, Ho Chi Minh City, Vietnam
}

Correspondence should be addressed to Zaher Mundher Yaseen; yaseen@tdtu.edu.vn

Received 30 November 2020; Revised 20 February 2021; Accepted 24 February 2021; Published 12 March 2021

Academic Editor: Baogui Xin

Copyright (c) 2021 Xinni Liu et al. This is an open access article distributed under the Creative Commons Attribution License, which permits unrestricted use, distribution, and reproduction in any medium, provided the original work is properly cited.

Deformation of ground during tunnelling projects is one of the complex issues that is required to be monitored carefully to avoid the unexpected damages and human losses. Accurate prediction of ground settlement (GS) is a crucial concern for tunnelling problems, and the adequate predictive model can be a vital tool for tunnel designers to simulate the ground settlement accurately. This study proposes relatively new hybrid artificial intelligence (AI) models to predict the ground settlement of earth pressure balance (EPB) shield tunnelling in the Bangkok MRTA project. The predictive models were various nature-inspired frameworks, such as differential evolution (DE), particle swarm optimization (PSO), genetic algorithm (GA), and ant colony optimizer (ACO) to tune the adaptive neuro-fuzzy inference system (ANFIS). To obtain the accurate and reliable results, the modeling procedure is established based on four different dataset scenarios including (i) preprocessed and normalized (PPN), (ii) preprocessed and nonnormalized (PPNN), (iii) non-preprocessed and normalized (NPN), and (iv) non-preprocessed and nonnormalized (NPNN) datasets. Results indicated that PPN dataset scenario significantly affected the prediction models in terms of their perdition accuracy. Among all the developed hybrid models, ANOFS-PSO model achieved the best predictability performance. In quantitative terms, PPN-ANFIS-PSO model attained the least root mean square error value (RMSE) of 7.98 and a correlation coefficient value (CC) of 0.83 . Overall, the attained results confirmed the superiority of the explored hybrid AI models as robust predictive model for ground settlement of earth pressure balance (EPB) shield tunnelling.

\section{Introduction}

The durability of underground excavation projects is normally dependent on accurate deformation prediction of the rock masses $[1,2]$. Currently, the construction of a tunnel in the urban locations with small construction depth is facing a significant increase in growth in the complex geological formations and there is increase in risk conditions by external loading from the building [3]. However, the increase of budge and construction delay may exist when these conditions cannot be acknowledged before excavating the tunnel. Thus, to prevent project delays around the tunnel, a significant valid prediction is needed [4], particularly the structural failure and excessive deformation and structural failure that are forecasted by monitoring and data collection within the tunnel. Subsequent genuine and helpful actions are taken based on the feedback information [5]. Regardless of an increase in the experience and theoretical assessment obtained from the monitored data using several construction techniques, targeted and reliable techniques of available predictions are still absent [6]. The analytical and empirical techniques are not suitable for all geological cases because they cannot produce reliable results but only forecast a few numbers of geo-mechanical applying simplifications and parameters [4]. 
Despite the use of the earth pressure balance (EPB) shields over the years, the actual mechanism that governs shield-ground interaction is yet to be fully understood. The understanding of the EPB tunnelling-induced ground response mechanism is difficult as it requires both a reliable measurement of ground deformations in the field and the EPB shields' operational records. Few studies on EPB tunnelling are currently available but being that there has been a significant increase in the use of EPB tunnelling technique in recent times; especially in urban environments, there is a need for engineers to have a better understanding of the mechanisms of EPB tunnelling and how its parameters influence ground deformations. This will help to reduce the detrimental effects of this mechanism on the immediate surrounding.

Some of the engineering mechanics attributes of tunnel rock masses bothered on the failure and mechanism, meaning that engineers neither readily nor accurately forecasted because of the lack of clarity on the extent of rock mass support interaction, heterogeneity of the rock mass, and geotechnical environments before construction. Many years ago, some major studies had concentrated on the regularities and rock masses deformation and mechanism of ground surface settlements according to the in situ test data collected from the past projects and accumulated experience that have detected the durability of the tunnels.

There are three kinds of techniques, including the artificial intelligence and analytical and numerical methods that can be utilized during practice to evaluate the tunnel deformation. An analytical technique was performed on a shallow tunnel in the saturated ground according to [7] where they used two kinds of drainage conditions with and without full drainage at the ground-line interface. The results obtained showed that the solutions are narrowed to where ground deformations are small. Chou and Bobet [8] utilized 28 tunnels in determining the predictions from an analytical method in shallow tunnels on saturated ground. Based on this result, the difference between observation and predictions from real tunnels showed about $15 \%$ good agreement. Other different studies were carried out using the analytical methodologies [9-11].

Several AI techniques have been developed for addressing the challenges associated with rock and geotechnical engineering $[12,13]$. Sou-Sen and Hsien-Chuang [14] used an artificial neural network- (ANN-) based regression model for the prediction of the influence of the ground surface settlement through intense excavation. They utilized case data that were obtained from just concluded deep excavation projects in Taiwan to develop a model. These results showed that the ANN-based forecasted models that can justifiably forecast the location and magnitude of maximum ground surface settlement influenced through deep excavation. An et al. [15] suggested an evolutionary neural network (ENN) model to simulate the ground settlement. The model was developed according to the mechanism which enables every part of the network structure, such as the learning parameters and several hidden nodes that can be developed via the genetic algorithms. These results showed a better achievement for the prediction of the ground settlement. Neaupane and Adhikari [16] established the ANN model for predicting ground movement throughout the tunnels. The predicted surface settlement above a horizontal ground movement and tunnel can be as a result of a tunnel construction through the assistance of input parameters that can cause direct physical significance. These results showed the ability of the ANN model to achieve positive outcomes and fairly and successfully forecasted the desired goal. Cheng et al. [17] developed an evolutionary fuzzy neuro-inference system (EFNIS) in facilitating geotechnical expert in decision making. The EFNIM consist of three separate AI technologies, such as genetic algorithm (GA), neural network (NN), and fuzzy logic (FL). Two case studies were considered, estimating slurry-wall duration that includes a selection of retaining wall construction techniques and estimating slurry-wall duration. The outcomes showed that EFNIS has an increased capability for the geotechnical challenges over the other classical AI models when the two references were compared. Santos and Celestino [18] confirmed the ANN model functionality by analysing tunnel settlement instrumentation. This study was centred on a settlement above shotcrete-supported tunnels on West Extension that were excavated in tertiary sediment utilizing the sequential excavation technique. The study has shown that the ANN model predicted accurately. Lee and Akutagawa [19] reported the ANN method as a rapid displacement prediction by utilizing the outcomes of field measurement in the NATM tunnels. They gathered data for NATM tunnels built on a coarse ground and analysed them based on the major tunnelling parameters, including tunnel displacement, support condition, and geometry discovered during constructions. The outcomes proposed that the ANN model could forecast tunnel deformations at the last phase before construction with reasonable increase in the level of accuracy of some information. Yao et al. [20] reported a multistep-ahead prediction model according to the SVM model of the tunnel that surrounds the rock displacement prediction. They used shuffled complex evolution algorithm (SCEUA) through some exponential transformations in improving the training capacity of the SVM. The outcomes demonstrated that SVM is better than the classical ANN and indicated that SVM can be an effective and feasible multistep technique for a tunnel which surrounds the rock displacement prediction. Pourtaghi and Lotfollahi-Yaghin [21] introduced the method that was based on a combination between the ANN model and wavelet theory for the prediction of maximum surface settlement due to tunnelling. The simulation results showed a reduction in estimated error values which represented the capacity to increase the activity approximation ability and wherefore exhibited outstanding learning capability as compared to the other activation activities. Wang et al. [22] utilized an easy relevancy vector machine with a wsRVM to module tunnelling-induced ground surface settlement establishment. Several conditions that affect settlement were examined, such as shield operational, geological, and geometrical parameters. The outcomes indicated that the prediction model works perfectly and the extension of the predictions could give a mean of 
predicting uncertainty. Khamesi et al. [23] presented a numerical analysis, imperialistic competitive algorithm, and intelligent back analysis technique combining fuzzy systems to improve the capacity of intelligent back analysis in tunnelling. The results showed that PSO achieved the best parameters tuning performance while ICA showed great capacity for world searching on designed fuzzy system and it was seen that the fuzzy systems are equal to the inputs with given outputs for making back analysis feasible in a large worldwide space and to apply these methods to additional difficult engineering problems. Ahangari et al. [24] used new intelligent techniques in predicting subway settlement depended on gene expression programming (GEP) and ANFIS model in the settlement prediction. They obtained data from fifty-three tunnels all over the world, forty data sets were used in intelligent modeling, while thirteen data were utilized to evaluate its role. They deduced that the two intelligent techniques are approved to predict subway settlements. Hasanipanah et al. [25] revealed that hybrid ANNPSO can be used to predict maximum surface settlement resulted from tunnelling. The model was developed based on 143 datasets. The results showed that the suggested PSOANN model can predict maximum surface settlements by producing an increased level of accuracy when compared to the ANN results.

The potential of the ANFIS model for modeling settlement problems has been documented in the literature [26]. Results indicated an optimistic research trend using the capability of the ANFIS model. However, there are certain problems with the ANFIS model and these are related with the tuning of the parameters of the membership function [27]. This problem is incorporated with the learning process of the model that at the first place influence the prediction accuracy. As a matter of fact, AI models are subjective to hyperparameters tuning [28]. The new era of computer aid application is advanced to the exploration of the hybridization of AI models with various nature inspired optimization algorithms such as ant colony (ACO), particle swarm optimization (PSO), genetic algorithm (GA), and differential evolution (DE) [29-32], which can be used to train AI models and improve their performance in addressing both high-dimensional and nonlinear problems. The introduced optimization algorithms have been evidenced their capacity in optimizing ANFIS model for modeling diverse prediction problems [33-38]. The hybridization of ANFIS model with nature inspired optimization algorithms demonstrated a remarkable improvement for prediction process of several engineering applications [39-43]. Hence, the main goal of the hybridization is to attain a stable and reliable learning process [44-46].

This work is driven by the need to study the feasibility of relatively new hybrid models based on the hybridization of ANFIS model with four different nature-inspired algorithms which are ACO, PSO, GA, and DE for modeling ground settlement of earth pressure balance (EPB) shield tunnelling. To attain an accurate and reliable predictability performance, the modeling procedure is established based on four different dataset scenarios where the data preprocessing and data normalization are incorporated. A comprehensive analysis and assessment are conducted on the achieved results. Several limitations are recognized and reported for possible future research devotion.

\section{Underground Tunnel and Data Description}

In the current research, the underground dataset used for the modeling development is belonged to the Bangkok Subway project. The project consisted of twenty kilometers length of twin tunnels. The entire project was initiated with eight $\mathrm{EPB}$ shields. The geological feature of the project is Chao Praya delta plain with topological around 1-0.5 meters above the sea level. During the excavation of the project, large number of surface settlement markers and array "within 50 meters depth" were installed to measure the ground settlement (see Figure 1). There are several factors effecting the surface settlement including the geometry of the tunnel (depth of the tunnel and the distance from shaft), geological parameters (e.g., the groundwater table, geology at the crown, and invert of the tunnel) and shield operation parameters (rate of penetration, face pressure, pitching angle, percentage tail void grout filling, and tail void grouping pressure) [47]. This study considered 13 related parameters to build the prediction matrix of the proposed hybrid predictive models.

\section{Methodology Overview}

3.1. Adaptive Neuro-Fuzzy Inference System (ANFIS). Neuro-fuzzy (NF) set approach represents an integrative soft computing method comprising of neural networks for patterns recognition for the immediate environment and a human expert-dependent fuzzy inference system for making solutions and differentiating solutions in a special field $[48,49]$. The performance of these systems in decisions processing and explanation ought to mimic human-like expertise [50]. Additionally, the system has a fault tolerance capability that ensures the system will not be adversely affected during a deletion/amendment task. However, the applicability of soft computing approach is gaining momentum with the development of a firm foundation in various fields all around the world. Adaptive neuro-fuzzy inference system (ANFIS) was developed as a branch of the AI models whose mechanism of operation is based on Takagi-Sugeno fuzzy inference system [51]. ANFIS combines the advantages of neural networks and fuzzy logic in a single framework [52]. It is also equipped with a fuzzy inference system which can be trained; thus, it is taken as a system with better efficiency compared to systems with only a neuro-fuzzy system. This work used different input parameters and only one output $f$ interference system. The rules of the firstorder Sugeno fuzzy model [53] are as follows: $f_{1}, f_{2}, \ldots, f_{n}$, where $n$ is the highest number of rules [54]:

$$
\begin{aligned}
& \text { Rule \#1: if } X \text { is } A_{1} \text { and } Y \text { is } B_{1} \text {, then } f_{1}=p_{1} x+q_{1} y+r_{1} \text {, } \\
& \text { Rule \#2: if } X \text { is } A_{2} \text { and } Y \text { is } B_{2} \text {, then } f_{2}=p_{2} x+q_{2} y+r_{2} \text {, }
\end{aligned}
$$

where $A_{1}-A_{2}$ and $B_{1}-B_{2}$ are the membership functions for multiple inputs including $x$ and $y$. Figure 2 presents the main ANFIS model structure. 


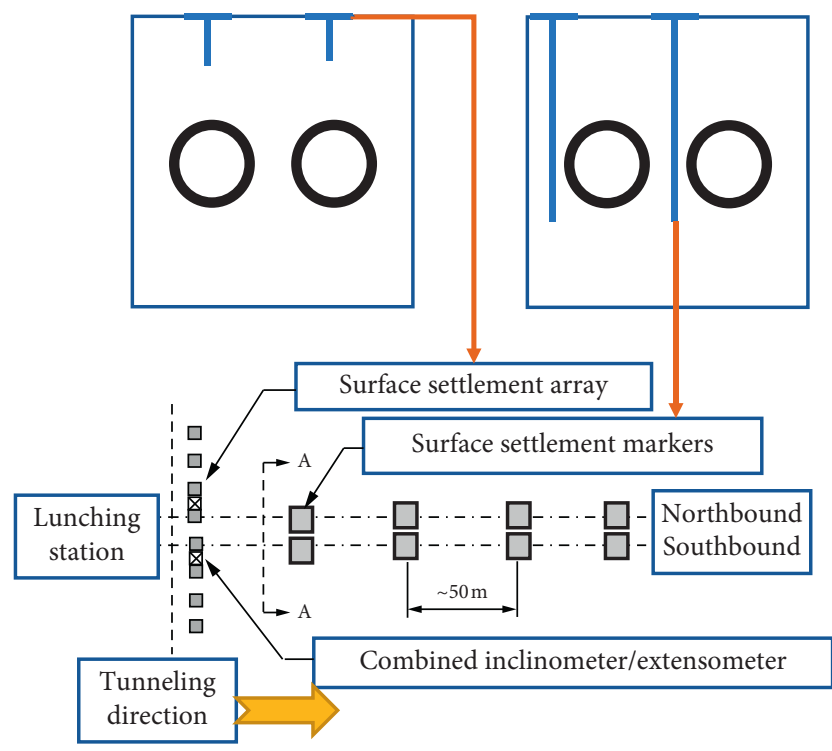

Figure 1: Typical instrumentation for ground surface settlement for the studied project.

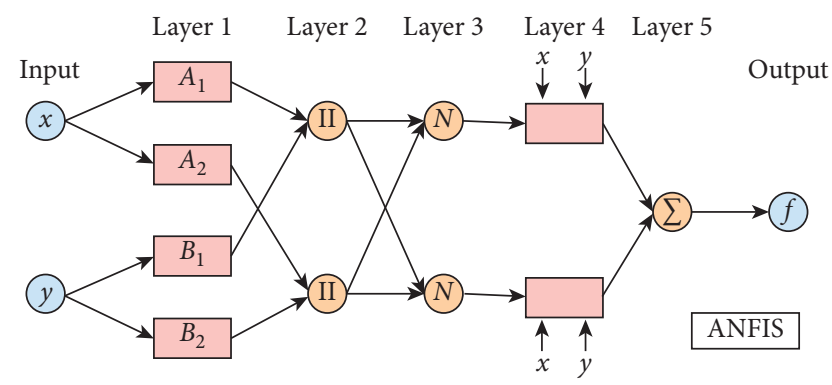

FIGURE 2: The structure of the adaptive neuro-fuzzy inference system model.

In an ANFIS model structure with multiple rules, several inputs, and one output, the nodes in the same layer perform the same role. The output of the $i^{\text {th }}$ node in the first layer is selected when the input membership functions (MFs) are contained in the first layer and the input values are delivered to the subsequent layer $[55,56]$. The generalized bell function must have the best nonlinear parameters categorization capability for it to be used. In the first layer, every node is squared to initiate the membership grades. The input parameters are translated into linguistic terms:

$$
O_{i}^{1}=\mu_{A_{i}}(x), \quad i=1,2,
$$

in which $x=$ input parameters of node $I, A_{i}=$ a linguistic term, and $O_{i}^{1}=$ the MF of $A_{i}$.

The output of the first layer is multiplied by the second layer (usually called a membership layer) to generate a new output. Each node in the second layer is considered a fixed node whose output depends on all the input values. The node $i$ in the 3rd layer computes the firing strength ratio of the rules, with the outputs taken as the normalized weights [57]. The values of the inference rules-based outputs are provided by the fourth layer; the overall inputs of the previous layers are combined in this layer before converting the classification results into the final output. The ANFIS structure is identified by the applied learning algorithm. The functional forward pass signal in this algorithm continues until the fifth layer (the defuzzification layer).

Least squares estimate was used to identify the consequent parameters. The error rates propagate backward in the backward pass while during the gradient descent, the premise parameters are updated. The ANFIS models' membership function was later tuned using several nature inspired optimization algorithms including PSO, GA, and DE optimization algorithms to ensure a minimum solar radiation prediction error.

3.2. Particle Swarm Optimization (PSO). PSO is a computational framework put together by Eberhart and Kennedy [58] in 1995 for continuous and discontinues decisionmaking processes. It was based on the natural behavior of living species such as the schooling of fishes when searching for food sources. Being that in the PSO, each particle in the population is considered as a potential solution, the PSO is regarded as a population search-based method. During an active search, the optimal solution found by every particle is varied in a multidimensional space until there are no more computation limitations to be addressed. In the PSO, the major problems encountered during swarm optimization are associated with the position of $N$ particles; this position is randomly assigned to the swarm in the D-dimensional space. Each solution in the swarm is associated with a position and each particle in the solution space is counted through a scoring function whose values explain the status of the problem. Several studies have applied PSO on several optimization problems [59], where it has been found that all the particles found the global best position in the solution space and achieved personal best positions. The new assigned position and velocity of the particles are updated by the following rules [60]:

$$
p=p+v
$$

with

$$
v=v+c_{1} \text {.rand }\left(p_{\text {best }}-p\right)+c_{2} \text {.rand. }\left(g_{\text {best }}-p\right),
$$

where $p$ and $v$ are the particle position and direction, $c_{1}$ is the local weight and $c_{2}$ is the global weight, $p_{\text {best }}$ and $g_{\text {best }}$ are the best positions for the particles and swarms, respectively, and rand is a random value. The operation of the PSO algorithm is depicted in Figure 3.

3.3. Genetic Algorithm (GA). The GA was first developed by [61] as an advanced optimization framework. As an AI framework, it uses vectors of 1's and 0's to represent complex structures. The GA was modeled after the concept of natural genetics as optimized functions are established by comparing 2 distinct approaches. The GA has a better capability of establishing global optimal solutions to huge combinatorial problem and this is its major advantage compared to 


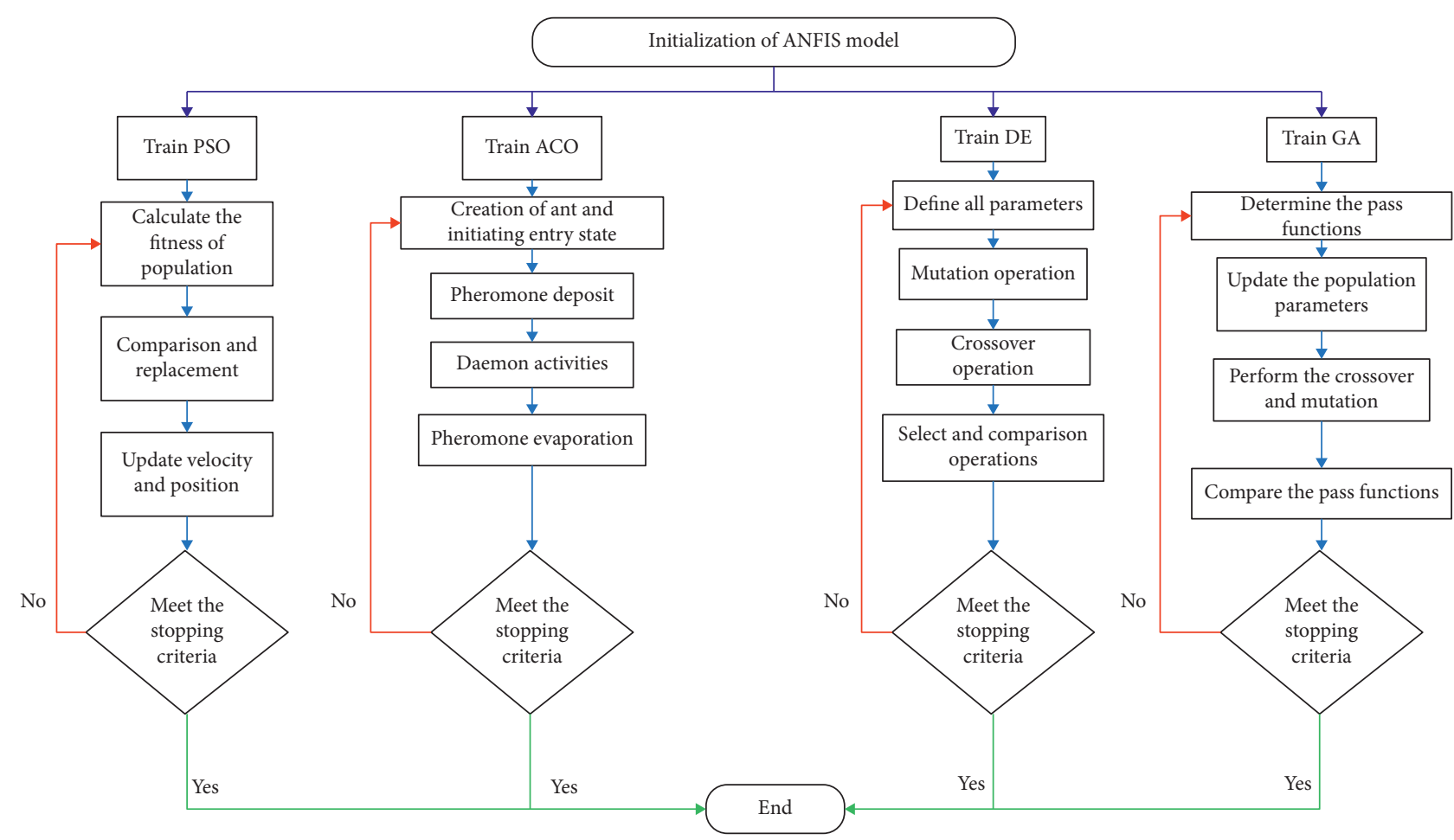

FIgURE 3: The flow chart of the proposed hybrid ANFIS models; the optimization procedure of each integrated nature inspired algorithm.

the other systems. Consequently, the GA is often used in multiobjective tasks optimization. The operation of the GA is similar to those of natural evolution processes; it relies on 3 operators which are selection, crossover, and mutation. The first optimization step using GA involves the evaluation of the fitness functions of the selected configuration (known as a chromosome). This step also involves sustaining a population of $\mathrm{M}$ solutions. If the annualized system cost of the evaluated chromosome is lower than the lowest known annualized system cost from the previous iterations, such a chromosome will be considered the optimal solution, and this would reduce the required number of problem iterations. Meanwhile, this optimum solution can also be exchanged with any better solution. Then, the crossover and mutation processes will be executed to select the best solution which will generate a new set of generations.

This process is repeated until an already specified convergence level is reached. Some precise parameters were used in the suggested GA model in this study; they are the size of the population, the rate of mutation, the crossover percentage, and the selection pressure (refer to Table 1). Figure 3 depicts the flow chart of the proposed GA.

3.4. Deferential Equation. The DE was developed as an intelligent framework for optimization tasks. Its operation mimics the basic optimized mutation, crossover, and selection operators. The DE relies on NP and D-dimensional parameter vectors because it is a parallel direct search method and is not impacted by minimization processes; hence, it is considered a population process when generating each generation $G$. In the $\mathrm{DE}$, an initial population vector is randomly selected to cover all the parameter spaces; all the random choices are assigned a uniform probability distribution. DE generates new parameter vectors based on the available preliminary solution by generating the weight difference between 2 population vectors and a $3^{\text {rd }}$ vector via a mutation operation as follows:

$$
x i, G=[x 1, i, G, x 2, i, G, \ldots, x n, i, G], \quad i=1,2, \ldots, k,
$$

where $x_{1}, G, i=1,2,3, \ldots, \mathrm{NP}$ are the generated mutant vectors using $v_{i, G+1}$, and $r_{1}, r_{2}$, and $r_{3}$ are arbitrarily selected numbers $\in[1,2,3, \ldots$,$] . . NP. Note that NP must have these$ values, with $i$ and $\mathrm{F}$ being actual that only differed from each other $\in[1,2,3, \ldots,] \ldots$ NP. The trial vector is established via a mixing process (crossover operation) that involves mixing the parameters of the mutated vector with the other parameters of the predetermined vectors as follows:

$$
x L j \leq x j, i, 1 \leq x U j,
$$

where $u_{i, G+1}$ is the trailer vector and $x_{i, G}$ is the target vector; rand $b(j)$ represents the $J^{\text {th }}$ uniform random evaluation $\epsilon \in[0.1], \operatorname{rnbr}(i)$ is a random value index $\in \in[1,2,3, \ldots, d]$, and CR is a user-specified crossover constant. Lastly, the trial vector that gives the lowest cost function value compared to the target vector is used during the selection operation as the target value in the subsequent generation. Being that each population must serve as the target vector, NP tasks are considered as a one-generation procedure. A study by [62] has provided a deep description of the standard DE. Table 1 presents the proposed DE models' structure while its flow chart is depicted in Figure 3. 
TABLE 1: The values of the models tuning parameters.

\begin{tabular}{|c|c|c|}
\hline Models & Parameters & Values \\
\hline \multirow{6}{*}{ Algorithm: PSO } & Global learning coefficient & 2 \\
\hline & Number of iterations & 1200 \\
\hline & Number of populations & 50 \\
\hline & Personal learning coefficient & 0.9 \\
\hline & Inertia weight damping ratio & 0.95 \\
\hline & Inertia weight & 1 \\
\hline \multirow{3}{*}{ Algorithm: DE } & Crossover probability & 0.1 \\
\hline & Lower bound of scaling factor & 0.3 \\
\hline & Upper bound of scaling factor & 0.7 \\
\hline \multirow{4}{*}{ Algorithm: ACO } & Number of populations & 50 \\
\hline & Number of iterations & 1200 \\
\hline & Deviation distance ratio & 1 \\
\hline & Intensification factor & 0.5 \\
\hline \multirow{8}{*}{ Algorithm: GA } & Selection pressure & 8 \\
\hline & Mutation percentage & 0.5 \\
\hline & Number of mutants & 50 \\
\hline & Mutation rate & 0.1 \\
\hline & Number of iterations & 1200 \\
\hline & Crossover percentage & 0.7 \\
\hline & Number of populations & 50 \\
\hline & Number of offspring & 35 \\
\hline \multirow{5}{*}{ ANFIS } & Train step size increase & 1.15 \\
\hline & Train step size decrease & 0.95 \\
\hline & Train initial step size & 0.01 \\
\hline & Train error goal & 0 \\
\hline & Train epoch & 500 \\
\hline
\end{tabular}

3.5. Ant Colony Optimization (ACO). ACO was put forward two decades ago by Dorigo [63] but has undergone several modifications since its development. ACO algorithms have a wide range of application due to their capability to solve both static and dynamic problems. Stignergy, a self-organizationenabling process, coordinates numerous activities in ant colonies; such activities include food hunting, labour partitioning, brood sorting, and cooperative transport in the ant colony. Ant colonies are known to contain simple individuals; yet, it is a complex but well-organized structure where all activities are coordinated by stignergy. Chemical deposits known as pheromone are deposited by the leading ants for the followers to trail while seeking the shortest route to food source. ACO relies on such procedure for establishing the optimal position in the solution space. The ants move in a forward and backward pattern and adopt a stepwise decision approach in finding the optimal solution to any problem [48-50]. Figure 3 shows the flowchart for ACO.

3.6. Modeling Development Scheme. The developed hybrid and standalone ANFIS models (ANFIS-PSO, ANFIS-DE, ANFIS-ACO, ANFIS-GA, and ANFIS) were constructed based on several geo-technical information. The information was used as numerical parameters to initiate the prediction matrix. Figure 4 presents the input/output parameters used in the current research. The dataset has a total of $49 \mathrm{ob}-$ servations and for the non-preprocessed datasets, the data were partitioned into $65 \%$ and $35 \%$ for training and testing purposes, respectively. For the preprocessed data, the modeling was executed with 38 observations based on the data partitioning percentage of $63 \%-35 \%$. Several statistical measures, plots, and error levels between the experimental and predicted ground settlements were reported during the testing phase. The prediction process was conducted based on several scenarios associated with the data processing including (i) preprocessed and normalized (PPN) dataset, (ii) preprocessed and nonnormalized (PPNN) dataset, (iii) non-preprocessed and normalized (NPN) dataset, and (iv) non-preprocessed and nonnormalized (NPNN) dataset "with total 20 modeling investigations model 1-model 20." The statistical performance of all the developed predictive models are reported in Tables $2-5$, respectively.

3.7. Modeling Performance Metrics. Several statistical metrics are computed to assess the predictability performance of the proposed predictive models including mean absolute error (MAE), root mean square error (RMSE), Legate and McCabe's index (LMI), PBIAS, Willmott's index (WI), correlation coefficient (CC), and relative root mean square error (RRMSE); these metrics were calculated, thus [64-68] 


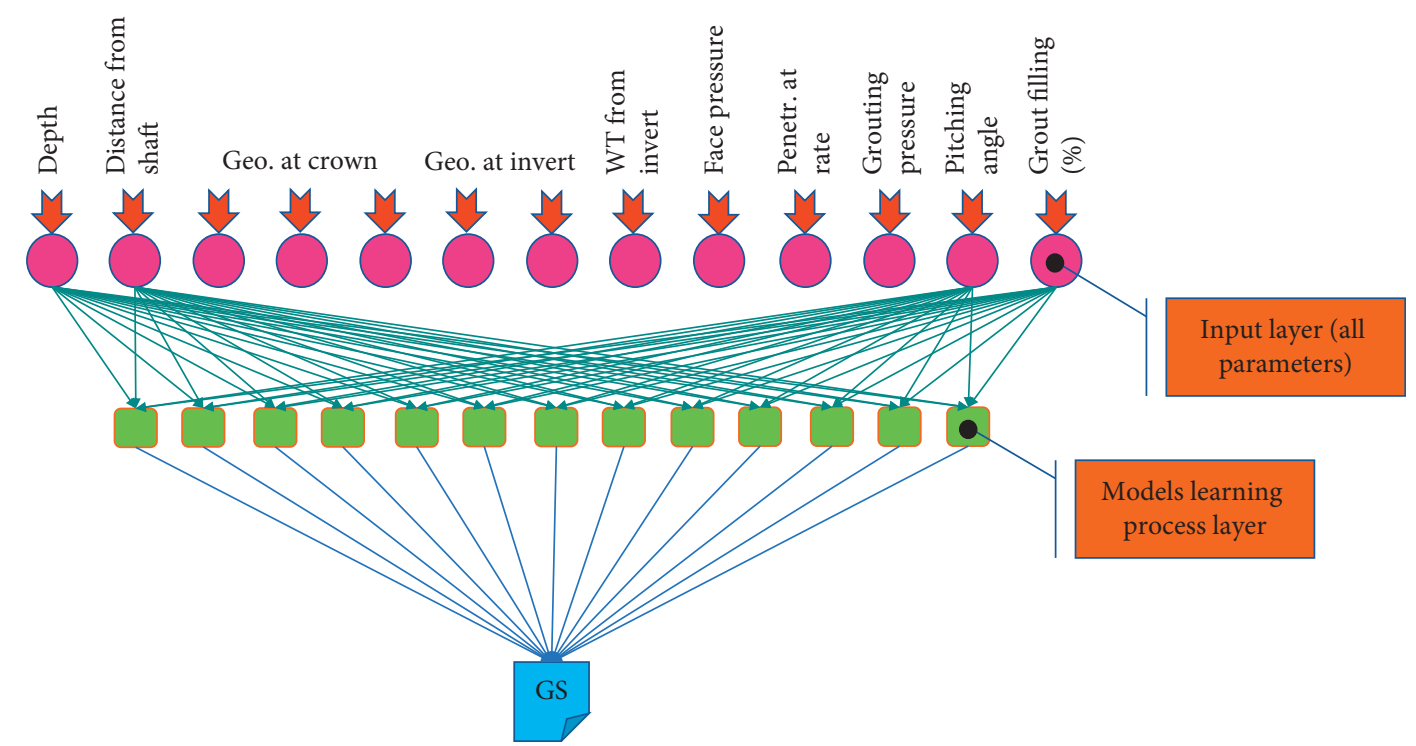

FIGURE 4: The related geo-science input parameters and the output ground settlement.

TABLE 2: The statistical evaluation of the developed hybrid and original ANFIS for the scenario of the preprocessed and normalized (PPN) dataset over the testing phase of the modeling.

\begin{tabular}{|c|c|c|c|c|c|c|c|}
\hline Models order & Scenario models & RMSE & MAE & LMI & $\mathrm{CC}$ & WI & SRMSE \\
\hline Model 1 & PPN-ANFIS & 11.57 & 8.77 & -0.06 & 0.74 & 0.76 & 41.89 \\
\hline Model 2 & PPN-ANFIS-PSO & 7.99 & 6.22 & 0.25 & 0.83 & 0.82 & 28.90 \\
\hline Model 3 & PPN-ANFIS-DE & 8.61 & 6.96 & 0.16 & 0.66 & 0.57 & 31.16 \\
\hline Model 4 & PPN-ANFIS-ACO & 8.98 & 7.49 & 0.10 & 0.73 & 0.36 & 32.49 \\
\hline Model 5 & PPN-ANFIS-GA & 9.23 & 7.19 & 0.13 & 0.76 & 0.75 & 33.39 \\
\hline
\end{tabular}

TABLE 3: The statistical evaluation of the developed hybrid and original ANFIS for the scenario of the preprocessed and nonnormalized (PPNN) dataset over the testing phase of the modeling.

\begin{tabular}{lccccccc}
\hline Models order & Scenario models & RMSE & MAE & LMI & CC & WI & SRMSE \\
\hline Model 6 & PPNN-ANFIS & 10.40 & 8.29 & 0.00 & 0.78 & 0.77 & 37.64 \\
Model 7 & PPNN-ANFIS-PSO & 8.78 & 6.60 & 0.20 & 0.80 & 0.78 \\
Model 8 & PPNN-ANFIS-DE & 9.08 & 7.51 & 0.09 & 0.41 & 0.60 \\
Model 9 & PPNN-ANFIS-ACO & 10.04 & 8.44 & -0.02 & -0.48 & 0.04 & 31.79 \\
Model 10 & PPNN-ANFIS-GA & 9.26 & 7.51 & 0.09 & 0.77 & 0.79 \\
\hline
\end{tabular}

TABLE 4: The statistical evaluation of the developed hybrid and original ANFIS for the scenario of the non-preprocessed and normalized (NPN) dataset over the testing phase of the modeling.

\begin{tabular}{|c|c|c|c|c|c|c|c|}
\hline Models order & Scenario models & RMSE & MAE & LMI & $\mathrm{CC}$ & WI & SRMSE \\
\hline Model 11 & NPN-ANFIS & 12.96 & 10.10 & -0.13 & 0.36 & 0.62 & 48.58 \\
\hline Model 12 & NPN-ANFIS-PSO & 10.82 & 8.14 & 0.09 & 0.29 & 0.59 & 40.54 \\
\hline Model 13 & NPN-ANFIS-DE & 8.99 & 7.44 & 0.17 & 0.49 & 0.59 & 33.70 \\
\hline Model 14 & NPN-ANFIS-ACO & 11.00 & 9.16 & -0.03 & -0.28 & 0.15 & 41.24 \\
\hline Model 15 & NPN-ANFIS-GA & 11.10 & 8.02 & 0.10 & 0.20 & 0.53 & 41.62 \\
\hline
\end{tabular}

TABLE 5: The statistical evaluation of the developed hybrid and original ANFIS for the scenario of the non-preprocessed and nonnormalized (NPNN) dataset over the testing phase of the modeling.

\begin{tabular}{|c|c|c|c|c|c|c|c|}
\hline Models order & Scenario models & RMSE & MAE & LMI & CC & WI & SRMSE \\
\hline Model 16 & NPNN-ANFIS & 14.62 & 11.81 & -0.32 & 0.30 & 0.56 & 54.79 \\
\hline Model 17 & NPNN-ANFIS-PSO & 10.07 & 8.11 & 0.09 & 0.38 & 0.63 & 37.73 \\
\hline Model 18 & NPNN-ANFIS-DE & 11.75 & 9.87 & -0.11 & -0.25 & 0.16 & 44.04 \\
\hline Model 19 & NPNN-ANFIS-ACO & 11.27 & 9.43 & -0.06 & -0.42 & 0.09 & 42.24 \\
\hline Model 20 & NPNN-ANFIS-GA & 10.67 & 8.55 & 0.04 & 0.38 & 0.62 & 40.01 \\
\hline
\end{tabular}




$$
\begin{aligned}
& \mathrm{RMSE}=\sqrt{\frac{1}{N_{s}} \sum_{j=1}^{N_{s}}\left((\mathrm{GS})_{\exp }-(\mathrm{GS})_{\text {Sim }}\right)^{2}}, \\
& \mathrm{MAE}=\frac{1}{N_{s}} \sum_{j=1}^{N_{s}}\left|(G S)_{\exp }-(\mathrm{GS})_{\operatorname{Sim}}\right|, \\
& \mathrm{LMI}=1-\left[\frac{\sum_{i=1}^{N_{s}}\left|(\mathrm{GS})_{\exp }-(\mathrm{GS})_{\operatorname{Sim}}\right|}{\sum_{i=1}^{N_{s}}\left|(\mathrm{GS})_{\exp }-\overline{(\mathrm{GS})_{\exp }}\right|}\right] \text {, } \\
& t \\
& \mathrm{CC}=\frac{\sum_{j=1}^{N_{s}}\left((\mathrm{GS})_{\exp }-\overline{(\mathrm{GS})_{\exp }}\right)\left((\mathrm{GS})_{\operatorname{Sim}}-\overline{(\mathrm{GS})_{\operatorname{Sim}}}\right)}{\sqrt{\sum_{j=1}^{N_{s}}\left((\mathrm{GS})_{\exp }-\overline{(\mathrm{GS})_{\exp }}\right)^{2} \sum_{j=1}^{N_{T}}\left((\mathrm{GS})_{\operatorname{Sim}}-\overline{(\mathrm{GS})_{\operatorname{Sim}}}\right)^{2}}}, \\
& \mathrm{WI}=1-\left[\frac{\sum_{i=1}^{N_{s}}\left((\mathrm{GS})_{\exp }-(\mathrm{GS})_{\operatorname{Sim}}\right)^{2}}{\sum_{i=1}^{N_{s}}\left(\left|(\mathrm{GS})_{\operatorname{Sim}}-\overline{(\mathrm{GS})_{\exp }}\right|+\left|(\mathrm{GS})_{\exp }-\overline{(\mathrm{GS})_{\exp }}\right|\right)^{2}}\right], \\
& \text { SRMSE }=\frac{\sqrt{\left(1 / N_{s}\right) \sum_{j=1}^{N_{s}}\left((\mathrm{GS})_{\exp }-(\mathrm{GS})_{\operatorname{Sim}}\right)^{2}}}{\overline{\left(d_{s} / D\right)_{\mathrm{Obs}}}},
\end{aligned}
$$

where the $(\mathrm{GS})_{\exp }$ and $(\mathrm{GS})_{\operatorname{Sim}}$ are the experimental and simulated ground settlements, $\overline{(\mathrm{GS})_{\exp }}$ and $\overline{(\mathrm{GS})_{\text {Sim }}}$ are their mean values, and $N_{s}$ is the sample size.

Among the proposed predictive models, superior model may be different in terms of different performance indices.
This weakness can be solved using a new index called mean performance (MP) which is the integrate of all employed indices. To compute the MP value of each predictive model, it is necessary to convert the indices to standardized form in the range of [0 1] as following equations [69]:

$$
\begin{aligned}
& \widehat{\operatorname{RMSE}} \mathrm{Model}(i)=\frac{\left(\mathrm{RMSE}_{\mathrm{All}}^{\max }-\mathrm{RMSE}_{\mathrm{Model}(i)}\right)}{\left(\mathrm{RMSE}_{\mathrm{All}}^{\max }-\mathrm{RMSE}_{\mathrm{All}}^{\min }\right)}, \\
& \widehat{\operatorname{MAE}}_{\text {Model }(i)}=\frac{\left(\mathrm{MAE}_{\mathrm{All}}^{\max }-\mathrm{MAE}_{\mathrm{Model}(i)}\right)}{\left(\mathrm{MAE}_{\mathrm{All}}^{\max }-\mathrm{MAE}_{\mathrm{All}}^{\min }\right)}, \\
& \widehat{\operatorname{LMI}}_{\operatorname{Model}(i)}=\frac{\left(\mathrm{LMI}_{\mathrm{Model}(i)}-\mathrm{LMI}_{\mathrm{All}}^{\mathrm{min}}\right)}{\left(\mathrm{LMI}_{\mathrm{All}}^{\max }-\mathrm{LMI}_{\mathrm{All}}^{\min }\right)}, \\
& \widehat{\mathrm{CC}}_{\mathrm{Model}(i)}=\frac{\left(\mathrm{CC}_{\mathrm{Model}(i)}-\mathrm{CC}_{\mathrm{All}}^{\min }\right)}{\left(\mathrm{CC}_{\mathrm{All}}^{\max }-\mathrm{CC}_{\mathrm{All}}^{\min }\right)}, \\
& \widehat{\mathrm{WI}}_{\mathrm{Model}(i)}=\frac{\left(\mathrm{WI}_{\mathrm{Model}(i)}-\mathrm{WI}_{\mathrm{All}}^{\min }\right)}{\left(\mathrm{WI}_{\mathrm{All}}^{\mathrm{max}}-\mathrm{WI}_{\mathrm{All}}^{\mathrm{min}}\right)}, \\
& \widehat{\operatorname{SRMSE}} \mathrm{Model}(i)=\frac{\left(\mathrm{SRMSE}_{\mathrm{All}}^{\max }-\mathrm{SRMSE}_{\mathrm{Model}(i)}\right)}{\left(\mathrm{SRMSE}_{\mathrm{All}}^{\max }-\mathrm{SRMSE}_{\mathrm{All}}^{\min }\right)},
\end{aligned}
$$

where $\widehat{\mathrm{RMS}} \mathrm{E}_{\operatorname{Model}(i)}, \widehat{\operatorname{MAE}}_{\operatorname{Model}(i)}, \widehat{\operatorname{LMI}}_{\operatorname{Model}(i)}, \widehat{\mathrm{CC}}_{\operatorname{Model}(i)}$, $\widehat{\mathrm{WI}}_{\text {Model }(i)}$, and SRMSE $\mathrm{E}_{\text {Model }(i)}$ are the standardized values of the utilized performance metrices of $i^{\text {th }}$ model (hybrid
ANFIS model), RMSE $\operatorname{mall}_{\text {max }}, \mathrm{MAE}_{\mathrm{All}}^{\max }, \mathrm{LMI}_{\mathrm{All}}^{\max }, \mathrm{CC}_{\mathrm{All}}^{\max }$, $\mathrm{WI}_{\mathrm{All}}^{\mathrm{max}}$, and $\mathrm{SRMSE}_{\mathrm{All}}^{\mathrm{max}}$ are the maximum values of indices among all predictive models while $\mathrm{RMSE}_{\mathrm{All}}^{\mathrm{min}}, \mathrm{MAE}_{\mathrm{All}}^{\mathrm{min}}$, 
$\mathrm{LMI}_{\mathrm{All}}^{\min }, \mathrm{CC}_{\mathrm{All}}^{\min }, \mathrm{WI}_{\mathrm{All}}^{\mathrm{min}}$, and $\mathrm{SRMSE}_{\mathrm{All}}^{\min }$ are the minimum ones.
Then, the MP metric of $i^{\text {th }}$ model (hybrid ANFIS) can be expressed mathematically as follows [70]:

$$
\mathrm{MP}_{\text {Model }(i)}=\frac{\left(\widehat{\mathrm{RMSE}} \mathrm{Model}(i)+\widehat{\mathrm{MAE}}_{\operatorname{Model}(i)}+\widehat{\mathrm{LMI}}_{\operatorname{Model}(i)}+\widehat{\mathrm{CC}}_{\operatorname{Model}(i)}+\widehat{\mathrm{WI}}_{\operatorname{Model}(i)}+\widehat{\mathrm{RMS}}_{\mathrm{Model}(i)}\right)}{6} .
$$

From equation (9) it is clear that the MP value of each model is the mean value of its standardized performance indices which is in the range of [0 1]. However, the superior model has the highest value of MP compared to other ones.

\section{Application Results and Analysis}

This study was initiated to study the feasibility of using different variants of hybrid ANFIS models for the prediction of the magnitude of settlement in soil. In nature, soil structure behavior is influenced by several morphological and external parameters such as structural loading. However, the main concern is to quantify the exact possible settlement can be experienced to maintain the build sustainability and safety. Soil is usually characterized by nonstationary varying and nonlinearly pattern with the constituents and their properties. Empirical formulas are not strong enough for the simulation of the relationships between soil settlement and the other related parameters; hence, the use of strong and reliable predictive tools for the determination of the effects of the independent parameters on the dependent parameters was proposed in this study. Indeed, these reliable predictive models can contribute to the geo-science practical implementations.

4.1. Statistical Evaluation of the Developed Hybrid Models. Based on the results in Table 2 (PPN scenario), the best prediction performance was attained for the ANFIS-PSO with minimal RMSE and MAE values (7.99 and 6.22) and maximum CC and WI values $(0.83$ and 0.82$)$. Table 3 reveals the modeling results of the second modeling scenario (PPNN). Based on the reported results in Table 3, the accurate prediction process was achieved using the same hybrid model developed for the first scenario (i.e., ANFISPSO). Model 7 indiacted minimum $\mathrm{RMSE} \approx 8.78$ and $\mathrm{MAE} \approx 6.6$, while the $\mathrm{CC} \approx 0.80$ and $\mathrm{WI} \approx 0.78$. Table 4 reported the third modeling scenario where the dataset was non-preprocessed and normalized. The hybridization of the differential evolution optimizer with ANFIS model attained the best predictability performance with computed statistical metrics $\quad(\mathrm{RMSE} \approx 8.99, \quad \mathrm{MAE} \approx 7.44, \quad \mathrm{LMI} \approx 0.17$, $\mathrm{CC} \approx 0.49$, WI $\approx 0.59$, and SRMSE $\approx 33.7$ ). The results of the last modeling scenario are tabulated in Table 5. The best prediction results were obtained using the ANFIS-PSO model with statistical performance metrics (RMSE $\approx 10.07$, $\mathrm{MAE} \approx 8.11, \mathrm{LMI} \approx 0.09, \mathrm{CC} \approx 0.38, \mathrm{WI} \approx 0.63$, and SRMSE $\approx 37.73)$. Generally, the variance of the prediction results achieved based on the developed classical and hybrid ANFIS models can be explained by the use of different learning processes, and $\mathrm{AI}$ models can attain during the train and test modeling phases.

The hybridization of ANFIS model with nature-inspired optimization algorithms yielded the maximum prediction accuracy as a result of the robust tuning of the membership function. In quantitative explanation and for the best input combination, these tables displayed the optimal prediction possibility using all the applied predictive models. Based on the results in Table 6, the minimal RMSE metric was achieved using PPN-ANFIS-PSO (RMSE $\approx 7.99$ ) followed by PPNNANFIS-PSO (RMSE $\approx 8.78$ ), NPN-ANFIS-DE $($ RMSE $\approx 8.99)$, and NPNN-ANFIS-PSO $($ RMSE $\approx 10.07)$.

4.2. Modeling Evaluation Based on Graphical Presentation. The proposed predictive models for the settlement quantification were examined using several graphical visualizations including mean performance index (MP), scatter plot, box plot, and Taylor diagram. Figure 5 demonstrates the MP of all the computed performance measures of the deployed predictive models and for all the examined scenarios over the testing modeling phase. Figures 5(a)-5(d) indicate similar prediction performance observed using the statistical metrics which are tabulated in Tables 2-5. ANFIS-PSO was the best predictive model for the $1^{\text {st }}, 2^{\text {nd }}$, and $4^{\text {th }}$ modeling scenarios with maximum $\mathrm{MP}=1.0$, whereas ANFIS-DE accomplished the best prediction results for the $3^{\text {rd }}$ modeling scenario non-preprocessed and normalized (NPN) dataset with $\mathrm{MP} \approx 0.99$. Due to the diverse prediction performances exemplified in Figure 5, the best predictive models were abstracted and validated with respect to the applied modeling scenarios (Figure 6). Based on the visualization in Figure 6, the various variations in modeling predictability were avoided. Figure 6 evidenced the feasibility of the $1^{\text {st }}$ modeling scenario which gave the best results with $\mathrm{MP}=1$ using the potential of the ANFIS-PSO model. This is clearly presenting the potential of the PSO tuning algorithm integrated with the standalone ANFIS for providing a robust and reliable predictive model for settlement prediction. The worst model was recognized for the non-preprocessed and nonnormalized dataset scenario with minimal $\mathrm{MP}=0.03$ using ANFIS-PSO.

The behavior of the best applied predictive models was drawn in the form of scatter plot in Figure 7. Scatter plot displays the variance between the experimental and predicted values of the settlement. The best predictive model was identified from the variance around the fitted line and the correlation coefficient magnitude. Figure 7 indicates that the preprocessed and normalized scenario using ANFISPSO model attained the best fit in accordance with the 
TABLE 6: Summary of the best statistical performance of the developed hybrid ANFIS for all the examined modeling scenarios over the testing modeling phase.

\begin{tabular}{lccccccc}
\hline Models order & Scenario models & RMSE & MAE & LMI & CC & WI & SRMSE \\
\hline Model 2 & PPN-ANFIS-PSO & 7.99 & 6.22 & 0.25 & 0.83 & 0.82 & 28.90 \\
Model 7 & PPNN-ANFIS-PSO & 8.78 & 6.60 & 0.20 & 0.80 & 0.78 & 31.79 \\
Model 13 & NPN-ANFIS-DE & 8.99 & 7.44 & 0.17 & 0.49 & 0.59 & 33.70 \\
Model 17 & NPNN-ANFIS-PSO & 10.07 & 8.11 & 0.09 & 0.38 & 0.63 & 37.73 \\
\hline
\end{tabular}

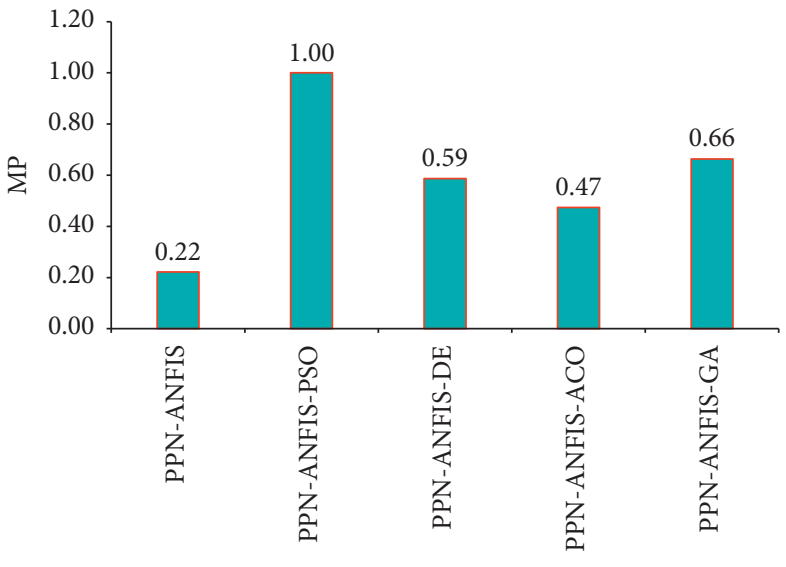

(a)

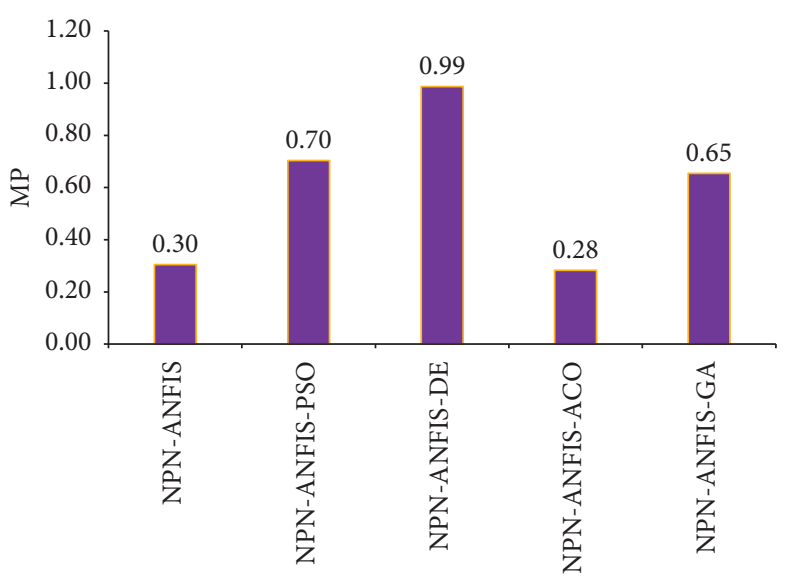

(c)

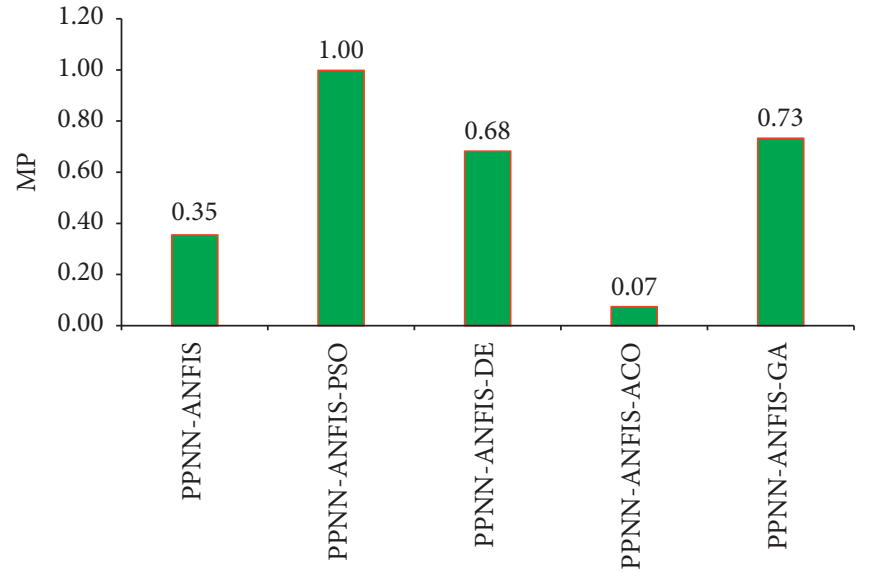

(b)

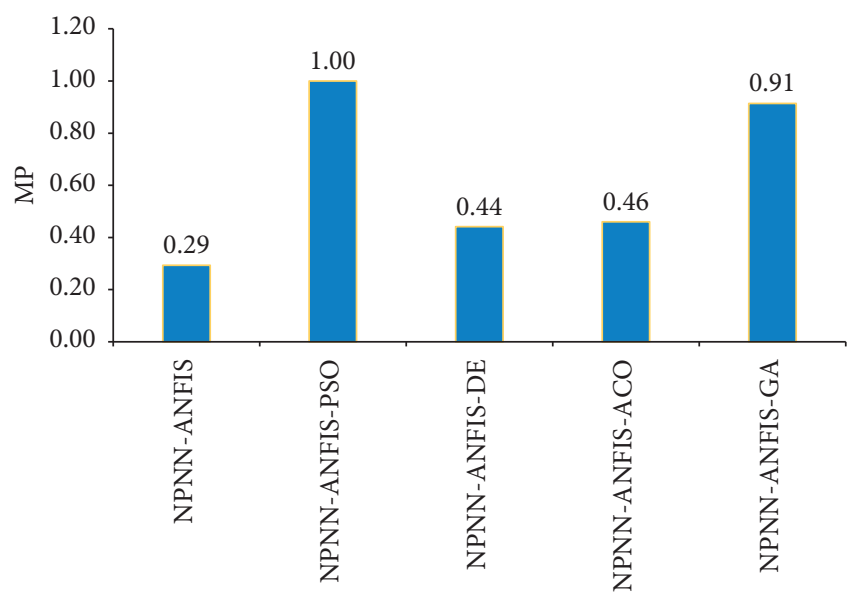

(d)

FIgURE 5: The mean performance index computed for the applied hybrid and classical ANFIS models over the test phase; (a) PPN scenario, (b) PPNN scenario, (c) NPN scenario, and (d) NPNN scenario.

determination coefficient scale $\left(R^{2}=0.69\right)$, whereas the worst indicator was observed for the $4^{\text {th }}$ scenario with $\left(R^{2}=0.14\right)$ using ANFIS-PSO model. Based on the reported determination coefficient, the models performed slightly with less correlation. However, this is clearly indicating the high stochasticity of the simulated surface settlement owing to the huge number of the associated parameters.

The box plot computations for the applied predictive models are displayed in Figure 8. The degree of the spread in the predicted data and quartiles are 25, 50, 75, and the interquartile range (IQR). Based on the magnitudes of the lower (Q25\%), median (Q50\%), and upper (Q75\%) quartiles, ANFIS-PSO with PPN scenario outperformed the other classical and hybrid ANFIS models. Based on the various statistical metrics and graphical representations, the proposed hybrid data-intelligence model is an excellent tool for predicting the settlement magnitudes.

The visualization of the 2-dimensional graphical examination of Taylor diagrams for the best prediction model (ANFIS-PSO) is shown in Figures 9(a) and 9(b) (processed and nonprocessed dataset, respectively). The figure presents a summary of the statistical performance of the actual and predicted tests in terms of the correlation coefficients, RMSE, and standard deviations. The Taylor curve is a graphical view of the similarity between the observed and predicted values. The results demonstrate that ANFIS-PSO 


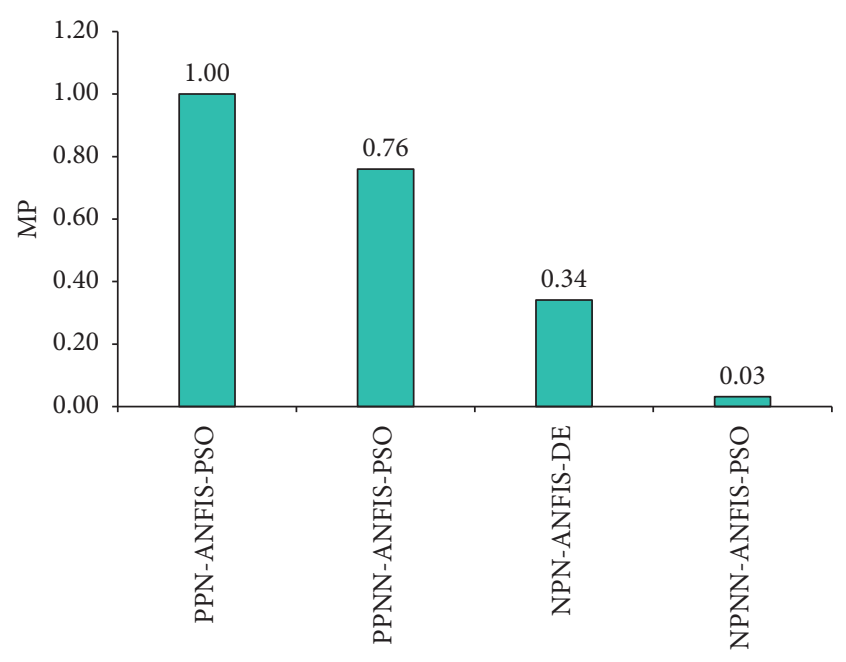

FIGURE 6: The best attained predictive models in accordance with the mean performance index over the testing phase.

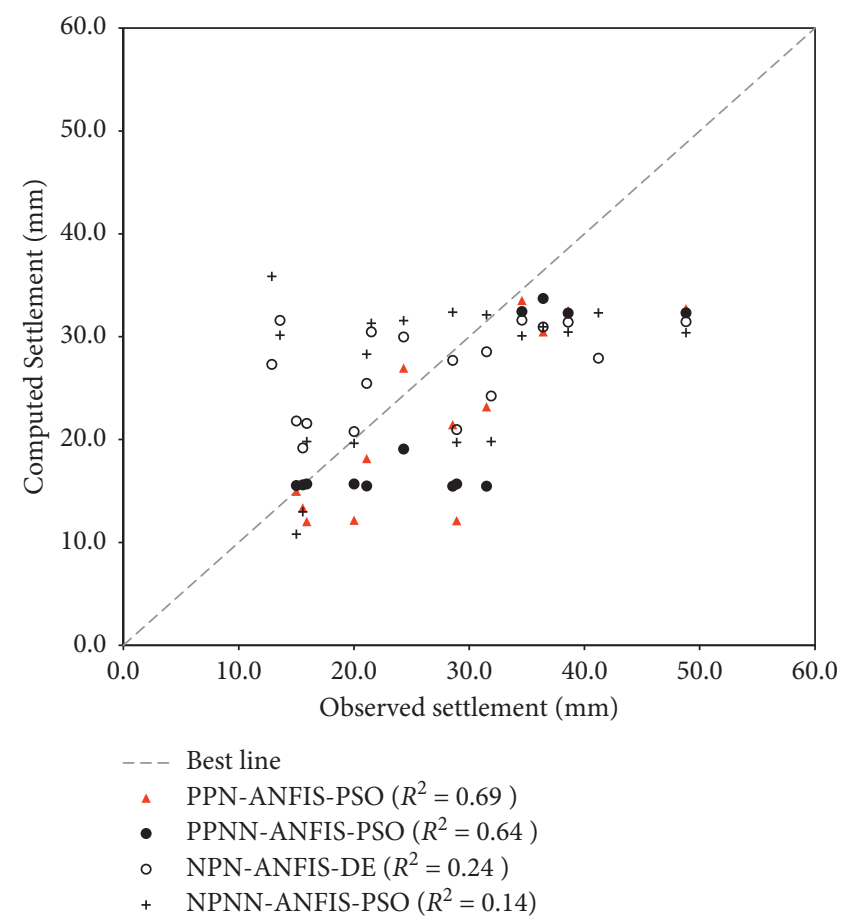

FIGURE 7: The variation between the observed and predicted settlement values over the testing phase and for the best predictive models.

using PPN scenario coordinated the nearest to the observed experimental tests. For the nonprocessed dataset, ANFIS-DE indicated the nearest coordination to the observed experimental tests.

4.3. Discussion and Possible Future Research Direction. In accordance to Figure 5, it was revealed that preprocessing the data by eliminating the redundant experimental test and scaling the data between (0-1) as normalized dataset could enhance the learning process of the applied hybrid predictive

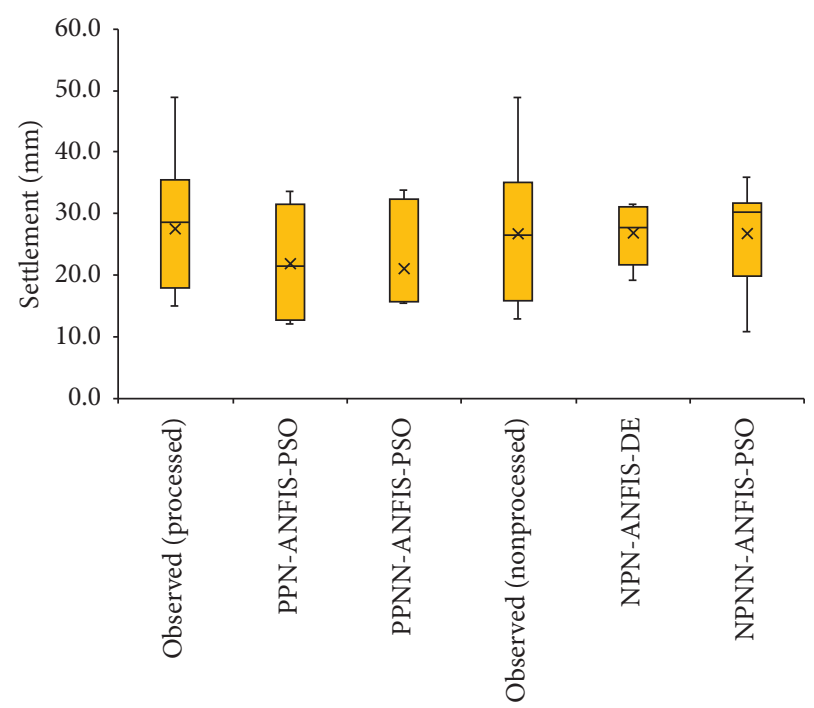

FIGURE 8: The box plots presentation of the observed and predicted settlement over the testing phase.

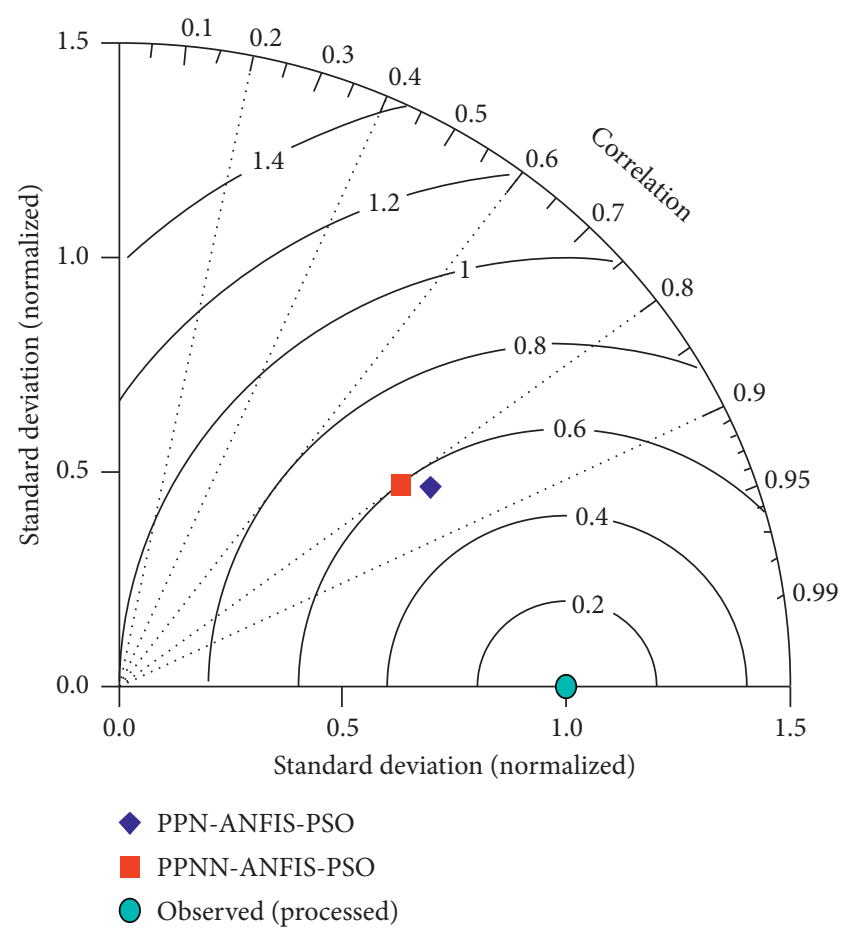

FIgURE 9: Normalized Taylor diagram visualization of the best applied predictive models over the testing phase.

models. This could be explained due to the exclusion of the vague attributes supplied to the prediction matrix. Hence, it is not always the case in providing more information attributes to fit data with higher accuracy. On the contrary, building the hybrid predictive model based on normal data information can offer insufficient learning process which leads to inaccurate prediction of result. The applications of AI models have been widely performed for several engineering problems and geo-technical is one of the distinguished research scopes experienced on the AI implications. 
Consequently, the development of more robust and reliable AI models was based on their newly advanced versions in the motivation of engineers, designers, and scientists. The proposed hybrid ANFIS-PSO model provided an efficient and flexible methodology for computing ground settlement compared to the classical AI standalone models. In addition, the current research introduced a newly fusion of technology to eliminate boundaries between the digital and engineering spheres. The breakthroughs of novel AI models, such as the proposed one, are changing the solving manners of engineering problems. Hence, the proposed hybrid ANFIS tool and other intelligent models can be used in future automated and semiautomated design processes.

In this study, several hybrid ANFIS models were developed to predict ground settlement of earth pressure balance (EPB) shield tunnelling. The models were constructed based on various modeling data preprocessing scenarios. In accordance with the attained prediction performance, the potential of the hybrid ANFIS model demonstrated an acceptable prediction accuracy using preprocessed and normalized dataset. However, the prediction capacity is varied from one hybrid model to another and this definitely evidenced the existence of the modeling uncertainty, that is, one of the major limitations of the current research. Another reason, the influence of the dataset span on the prediction performance. In addition, one of the major limitations of ANFIS model is that when a large number of inputs are applied that causing a dimensionality issues and computational expense. Therefore, an optimizer is required to reduce these problems. In contrary, those metaheuristic algorithms also have their own limitations, such as unstable convergence for the DE algorithms and time consumption for the GA, while the PSO suffers from partial optimism. All in all, it is impossible to say that there is one metaheuristic method which can compete with other methods in all possible discrete functions. Thus, based on the reported modeling limitations, the following possible future researches can be further studied: (i) inspecting the associated uncertainties, either modeling uncertainty or input parameters uncertainty; (ii) extending the span of the modeled dataset with other related studies from the literature to give more informative details of the input attributes; and (iii) the data modeling division, various data division percentages can be examined for better learning process of the established models; (iv) the achieved modeling results accuracy level can be further improved by using several other nature-inspired algorithms such as nomadic algorithm [71], equilibrium algorithm [72], arithmetic algorithm [73], and black window algorithm [74].

\section{Conclusion}

Among several side effects during tunnelling excavation and initiating, ground settlement is one of the essential processes to be measured and monitored to avoid any unexpected damages and human losses. In general, ground settlement is a complex issue as there are several geo-technical parameters influencing the soil deformation. Accurate and reliable prediction of ground settlement is very important for tunnelling project management and maintenance and thus an adequate intelligent predictive model can contribute to the basic knowledge of designing tunnels. The current research was devoted on the assessment of relatively new hybrid AI models to predict the ground settlement of EPB shield tunnelling. The data collected from tunnel project were established in Thailand in the name of Bangkok MRTA project. The developed hybrid and standalone ANFIS models (i.e., ANFIS-PSO, ANFIS-DE, ANFIS-ACO, ANFIS-GA, and ANFIS) were constructed based on several related parameters to predict the GS. For the purpose of obtaining accurate and reliable investigation, the modeling procedure was conducted based on four different dataset scenarios including (i) preprocessed and normalized (PPN), (ii) preprocessed and nonnormalized (PPNN), (iii) non-preprocessed and normalized (NPN), and (iv) non-preprocessed and nonnormalized (NPNN). Modelling results revealed the superiority of the first modeling scenario that preprocessed and normalized dataset were incorporated. PPN dataset scenario demonstrated a significant impact on the perdition accuracy of the proposed models. In addition, among all the applied models, the hybrid ANFIS-PSO model accomplished the best predictability performance. The PPN-ANFIS-PSO model achieved minimum $(\mathrm{RMSE}=7.98)$ and maximum $(\mathrm{CC}=0.83)$.

\section{Data Availability}

Data used in this study will be provided upon request.

\section{Conflicts of Interest}

The authors declare no conflicts of interest.

\section{References}

[1] J. Shen, M. Karakus, and C. Xu, "A comparative study for empirical equations in estimating deformation modulus of rock masses," Tunnelling and Underground Space Technology, vol. 32, pp. 245-250, 2012.

[2] A. Kayabasi, C. Gokceoglu, and M. Ercanoglu, "Estimating the deformation modulus of rock masses: a comparative study," International Journal of Rock Mechanics and Mining Sciences, vol. 40, no. 1, pp. 55-63, 2003.

[3] G. Barla and S. Pelizza, "TBM tunnelling in difficult ground conditions," in ISRM International Symposium, Melbourne, Australia, November 2000.

[4] S. Mahdevari, S. R. Torabi, and M. Monjezi, "Application of artificial intelligence algorithms in predicting tunnel convergence to avoid TBM jamming phenomenon," International Journal of Rock Mechanics and Mining Sciences, vol. 55, pp. 33-44, 2012.

[5] A. N. Jiang, S. Y. Wang, and S. L. Tang, "Feedback analysis of tunnel construction using a hybrid arithmetic based on Support Vector Machine and Particle Swarm Optimisation," Automation in Construction, vol. 20, no. 4, pp. 482-489, 2011.

[6] L. Cantieni, G. Anagnostou, and R. Hug, "Interpretation of core extrusion measurements when tunnelling through 
squeezing ground," Rock Mechanics and Rock Engineering, vol. 44, no. 6, p. 641, 2011.

[7] A. Bobet, "Analytical solutions for shallow tunnels in saturated ground," Journal of Engineering Mechanics, vol. 127, no. 12 , pp. 1258-1266, 2001.

[8] W.-I. Chou and A. Bobet, "Predictions of ground deformations in shallow tunnels in clay," Tunnelling and Underground Space Technology, vol. 17, no. 1, pp. 3-19, 2002.

[9] K.-H. Park, "Analytical solution for tunnelling-induced ground movement in clays," Tunnelling and Underground Space Technology, vol. 20, no. 3, pp. 249-261, 2005.

[10] M.-W. Gui and S.-L. Chen, "Estimation of transverse ground surface settlement induced by DOT shield tunneling," Tunnelling and Underground Space Technology, vol. 33, pp. 119$130,2013$.

[11] J. Xu and Y. Xu, "Grey correlation-hierarchical analysis for metro-caused settlement," Environmental Earth Sciences, vol. 64, no. 5, pp. 1249-1256, 2011.

[12] S. Yagiz, C. Gokceoglu, E. Sezer, and S. Iplikci, “Application of two non-linear prediction tools to the estimation of tunnel boring machine performance," Engineering Applications of Artificial Intelligence, vol. 22, no. 4-5, pp. 808-814, 2009.

[13] E. Ghasemi, "Particle swarm optimization approach for forecasting backbreak induced by bench blasting," Neural Computing and Applications, vol. 28, no. 7, pp. 1855-1862, 2017.

[14] L. Sou-Sen and L. Hsien-Chuang, "Neural-network-based regression model of ground surface settlement induced by deep excavation," Automation in Construction, vol. 13, no. 3, pp. 279-289, 2004.

[15] H. An, J. Sun, and X. Hu, "Study on intelligent method of prediction by small samples for ground settlement in shield tunnelling," in Proceedings of the 30th ITA-AITES World Tunnel Congress C, H. An, J. Sun, and X. Hu, Eds., Istanbul, Turkey, May 2004.

[16] K. M. Neaupane and N. R. Adhikari, "Prediction of tunnelinginduced ground movement with the multi-layer perceptron," Tunnelling and Underground Space Technology, vol. 21, no. 2, pp. 151-159, 2006.

[17] M.-Y. Cheng, H.-C. Tsai, C.-H. Ko, and W.-T. Chang, "Evolutionary fuzzy neural inference system for decision making in geotechnical engineering," Journal of Computing in Civil Engineering, vol. 22, no. 4, pp. 272-280, 2008.

[18] O. J. Santos Jr and T. B. Celestino, "Artificial neural networks analysis of São Paulo subway tunnel settlement data," Tunnelling and Underground Space Technology, vol. 23, no. 5, pp. 481-491, 2008.

[19] J.-H. Lee and S. Akutagawa, "Quick prediction of tunnel displacements using Artificial Neural Network and field measurement results," International Journal of the JCRM, vol. 5, no. 2, pp. 53-62, 2009.

[20] B.-Z. Yao, C.-Y. Yang, J.-B. Yao, and J. Sun, "Tunnel surrounding rock displacement prediction using support vector machine," International Journal of Computational Intelligence Systems, vol. 3, no. 6, pp. 843-852, 2010.

[21] A. Pourtaghi and M. A. Lotfollahi-Yaghin, "Wavenet ability assessment in comparison to ANN for predicting the maximum surface settlement caused by tunneling," Tunnelling and Underground Space Technology, vol. 28, pp. 257-271, 2012.

[22] F. Wang, B. Gou, and Y. Qin, "Modeling tunneling-induced ground surface settlement development using a wavelet smooth relevance vector machine," Computers and Geotechnics, vol. 54, pp. 125-132, 2013.
[23] H. Khamesi, S. R. Torabi, H. Mirzaei-Nasirabad, and Z. Ghadiri, "Improving the performance of intelligent back analysis for tunneling using optimized fuzzy systems: case study of the Karaj Subway Line 2 in Iran," Journal of Computing in Civil Engineering, vol. 29, no. 6, p. 5014010, 2014.

[24] K. Ahangari, S. R. Moeinossadat, and D. Behnia, "Estimation of tunnelling-induced settlement by modern intelligent methods," Soils and Foundations, vol. 55, no. 4, pp. 737-748, 2015.

[25] M. Hasanipanah, M. Noorian-Bidgoli, D. Jahed Armaghani, and H. Khamesi, "Feasibility of PSO-ANN model for predicting surface settlement caused by tunneling," Engineering with Computers, vol. 32, no. 4, pp. 705-715, 2016.

[26] A. F. Cabalar, A. Cevik, and C. Gokceoglu, "Some applications of adaptive neuro-fuzzy inference system (ANFIS) in geotechnical engineering," Computers and Geotechnics, vol. 40, pp. 14-33, 2012.

[27] D. Karaboga and E. Kaya, "Training ANFIS by using an adaptive and hybrid artificial bee colony algorithm (aABC) for the identification of nonlinear static systems," Arabian Journal for Science and Engineering, vol. 44, no. 4, pp. 35313547, 2019.

[28] A. A. Al-Musawi, A. A. H. Alwanas, S. Q. Salih, Z. H. Ali, M. T. Tran, and Z. M. Yaseen, "Shear strength of SFRCB without stirrups simulation: implementation of hybrid artificial intelligence model," Engineering with Computers, vol. 36, no. $1,2020$.

[29] M. A. Jayaram, M. C. Nataraja, and C. N. Ravi Kumar, "Design of high performance concrete mixes through particle swarm optimization," Journal of Intelligent Systems, vol. 19, no. 3, pp. 249-264, 2010.

[30] M. Flint, S. Grünewald, and J. Coenders, "Ant colony optimization for ultra high performance concrete structures," Designing and Building with UHPFRC, 2013.

[31] G. Quaranta, A. Fiore, and G. C. Marano, "Optimum design of prestressed concrete beams using constrained differential evolution algorithm," Structural and Multidisciplinary Optimization, vol. 49, no. 3, 2014.

[32] C. a. Coello Coello, a. D. Christiansen, and F. S. Hernández, "A simple genetic algorithm for the design of reinforced concrete beams," Engineering with Computers, vol. 13, no. 4, 1997.

[33] K. Elbaz, S.-L. Shen, W.-J. Sun, Z.-Y. Yin, and A. Zhou, "Prediction model of shield performance during tunneling via incorporating improved particle swarm optimization into ANFIS," IEEE Access, vol. 8, pp. 39659-39671, 2020.

[34] P. Zhang, Z.-Y. Yin, Y.-F. Jin, and T. H. T. Chan, "A novel hybrid surrogate intelligent model for creep index prediction based on particle swarm optimization and random forest," Engineering Geology, vol. 265, Article ID 105328, 2020.

[35] M. Babanezhad, I. Behroyan, A. T. Nakhjiri, A. Marjani, M. Rezakazemi, and S. Shirazian, "High-performance hybrid modeling chemical reactors using differential evolution based fuzzy inference system," Scientific Reports, vol. 10, no. 1, pp. 1-11, 2020.

[36] H. Jing, H. N. Rad, M. Hasanipanah, D. J. Armaghani, and S. N. Qasem, "Design and implementation of a new tuned hybrid intelligent model to predict the uniaxial compressive strength of the rock using SFS-ANFIS," Engineering with Computers, pp. 1-18, 2020.

[37] L. Penghui, A. A. Ewees, B. H. Beyaztas et al., "Metaheuristic optimization algorithms hybridized with artificial intelligence model for soil temperature prediction: novel model," IEEE Access, vol. 8, pp. 51884-51904, 2020. 
[38] S. Zhang, X.-N. Bui, N.-T. Trung, H. Nguyen, and H.-B. Bui, "Prediction of rock size distribution in mine bench blasting using a novel ant colony optimization-based boosted regression tree technique," Natural Resources Research, vol. 29, no. 2, pp. 867-886, 2020.

[39] Q.-T. Bui, M. Van Pham, Q.-H. Nguyen, L. X. Nguyen, and H. M. Pham, "Whale Optimization Algorithm and Adaptive Neuro-Fuzzy Inference System: a hybrid method for feature selection and land pattern classification," International Journal of Remote Sensing, pp. 1-16, 2019.

[40] A. Jaafari, E. K. Zenner, M. Panahi, and H. Shahabi, "Hybrid artificial intelligence models based on a neuro-fuzzy system and metaheuristic optimization algorithms for spatial prediction of wildfire probability," Agricultural and Forest Meteorology, vol. 266-267, pp. 198-207, 2019.

[41] K. Elbaz, S.-L. Shen, A. Zhou, D.-J. Yuan, and Y.-S. Xu, "Optimization of EPB shield performance with adaptive neuro-fuzzy inference system and genetic algorithm," Applied Sciences, vol. 9, no. 4, p. 780, 2019.

[42] P. A. Sari, M. Suhatril, N. Osman et al., "Developing a hybrid adoptive neuro-fuzzy inference system in predicting safety of factors of slopes subjected to surface eco-protection techniques," Engineering with Computers, vol. 36, no. 6, pp. 1-8, 2019.

[43] Z. M. Yaseen, W. H. Melini, H. W. Mohtar et al., "Implementation of Univariate Paradigm for streamflow simulation using hybrid data-driven model: case study in tropical region," IEEE Access, vol. 7, 2019.

[44] M. J. Varnamkhasti, "A hybrid of adaptive neuro-fuzzy inference system and genetic algorithm," Journal of Intelligent \& Fuzzy Systems, vol. 25, no. 3, pp. 793-796, 2013.

[45] B. Mohammad and J. Varnamkhasti, "ANFISGA-adaptive neuro-fuzzy inference system genetic algorithm," Global Journal of Computer Science and Technology, vol. 11, no. 1, 2011.

[46] Z. Yuan, L. N. Wang, and X. Ji, "Prediction of concrete compressive strength: research on hybrid models genetic based algorithms and ANFIS," Advances in Engineering Software, 2014.

[47] S. Suwansawat and H. H. Einstein, "Artificial Neural Networks for Predicting the Maximum Surface Settlement Caused by EPB Shield Tunneling," Tunnelling and Underground Space Technology, 2006.

[48] J. S. R. Jang and C. T. Sun, "Neuro-fuzzy modeling and control," in Proceedings of the IEEE, vol. 83, no. 3, pp. 378-406, 1995.

[49] L. Rutkowski, K. Cpałka, R. Nowicki, A. Pokropińska, and R. Scherer, "Neuro-fuzzy systems," in Proceedings of the Computational Complexity: Theory, Techniques, and Applications, 2012.

[50] J. Vieira, F. Dias, and A. Mota, "Neuro-fuzzy systems: a survey," in Proceedings of the 5th WSEAS NNA International Conference on Neural Networks and Applications, Udine, Italy, March 2004.

[51] J. S. R. Jang, "ANFIS: adaptive-network-based fuzzy inference system," IEEE Transactions on Systems, Man and Cybernetics, 1993.

[52] N. Walia, H. Singh, and A. Sharma, "ANFIS: adaptive neurofuzzy inference system-a survey," International Journal of Computer Applications, 2015.

[53] M. Sugeno and G. T. Kang, "Structure identification of fuzzy model," Fuzzy Sets and Systems, vol. 28, no. 1, pp. 15-33, 1988.

[54] V. Nikolić, D. Petković, S. Shamshirband, and Ž. Ćojbašić, "Adaptive neuro-fuzzy estimation of diffuser effects on wind turbine performance," Energy, vol. 89, no. C, pp. 324-333, 2015.

[55] H. Tao, A. A. Ewees, A. O. Al-Sulttani et al., "Global solar radiation prediction over North Dakota using air temperature: development of novel hybrid intelligence model," Energy Reports, vol. 7, pp. 136-157, 2021.

[56] S. Maroufpoor, E. Maroufpoor, O. Bozorg-Haddad, J. Shiri, and Z. Mundher Yaseen, "Soil moisture simulation using hybrid artificial intelligent model: hybridization of adaptive neuro fuzzy inference system with grey wolf optimizer algorithm," Journal of Hydrology, vol. 575, 2019.

[57] G. Landeras, J. J. López, O. Kisi, and J. Shiri, "Comparison of gene expression programming with neuro-fuzzy and neural network computing techniques in estimating daily incoming solar radiation in the Basque Country (Northern Spain)," Energy Conversion and Management, 2012.

[58] J. Kennedy and R. Eberhart, "A new optimizer using particles swarm theory," in Proceedings of the Sixth International Symposium on Micro Machine and Human Science, Nagoya, Japan, October 1995.

[59] M. A. Mohandes, "Modeling global solar radiation using Particle Swarm Optimization (PSO)," Solar Energy, vol. 86, no. 11, pp. 3137-3145, 2012.

[60] J. P. S. Catalão, H. M. I. Pousinho, and V. M. F. Mendes, "Hybrid wavelet-PSO-ANFIS approach for short-term electricity prices forecasting," IEEE Transactions on Power Systems, 2011.

[61] J. H. Holland, "Adaptation in natural and artificial systems: an introductory analysis with applications to biology," Control and Artificial Intelligence, MIT Press, Cambridge, MA, USA, 1975.

[62] R. Storn and K. Price, "Differential evolution-a simple and efficient heuristic for global optimization over continuous spaces," Journal of Global Optimization, 1997.

[63] M. Dorigo and G. Di Caro, "Ant colony optimization: a new meta-heuristic," in Proceedings of the 1999 Congress on Evolutionary Computation, CEC 1999, Washington, DC, USA, July 1999.

[64] A. A. H. Alwanas, A. A. Al-Musawi, S. Q. Salih, H. Tao, M. Ali, and Z. M. Yaseen, "Load-carrying capacity and mode failure simulation of beam-column joint connection: application of self-tuning machine learning model," Engineering Structures, vol. 194, 2019.

[65] B. Keshtegar, M. Bagheri, and Z. M. Yaseen, "Shear strength of steel fiber-unconfined reinforced concrete beam simulation: application of novel intelligent model," Composite Structures, vol. 212, 2019.

[66] Z. M. Yaseen, S. M. Awadh, A. Sharafati, and S. Shahid, "Complementary data-intelligence model for river flow simulation," Journal of Hydrology, vol. 567, 2018.

[67] Z. Yaseen, M. Ehteram, A. Sharafati, S. Shahid, N. Al-Ansari, and A. El-Shafie, "The integration of nature-inspired algorithms with least square support vector regression models: application to modeling river dissolved oxygen concentration," Water, vol. 10, no. 9, p. 1124, 2018.

[68] Z. A. Al-Sudani, S. Q. Salih, and Z. M. Yaseen, "Development of multivariate adaptive regression spline integrated with differential evolution model for streamflow simulation," Journal of Hydrology, vol. 573, pp. 1-12, 2019.

[69] A. Sharafati, K. Khosravi, P. Khosravinia et al., "The potential of novel data mining models for global solar radiation prediction," International Journal of Environmental Science and Technology, vol. 16, no. 11, pp. 7147-7164, 2019. 
[70] A. Sharafati, R. Yasa, and H. M. Azamathulla, "Assessment of stochastic approaches in prediction of wave-induced pipeline scour depth," Journal of Pipeline Systems Engineering and Practice, vol. 9, no. 4, Article ID 04018024, 2018.

[71] S. Q. Salih and A. A. Alsewari, "A new algorithm for normal and large-scale optimization problems: nomadic people optimizer," Neural Computing and Applications, vol. 32, no. 14, pp. 1-28, 2019.

[72] A. Faramarzi, M. Heidarinejad, B. Stephens, and S. Mirjalili, "Equilibrium optimizer: a novel optimization algorithm," Knowledge-Based Systems, vol. 191, Article ID 105190, 2020.

[73] L. Abualigah, A. Diabat, S. Mirjalili, M. Abd Elaziz, and A. H. Gandomi, "The arithmetic optimization algorithm," Computer Methods in Applied Mechanics and Engineering, vol. 376, p. 113609, 2021.

[74] V. Hayyolalam and A. A. P. Kazem, "Black widow optimization algorithm: a novel meta-heuristic approach for solving engineering optimization problems," Engineering Applications of Artificial Intelligence, vol. 87, Article ID 103249, 2020. 